\title{
An Exploratory Study to Assess Factors Associated with Adherence to Dots Therapy Among Patients Reporting at Selected Dots Centers in Punjab.
}

\author{
Tarandeep Kaur ${ }^{1}$, Deepak Sethi ${ }^{2}$ \\ ${ }^{I}$ M.Sc Nursing (Medical Surgical Nursing) Student, Saraswati Nursing Institute, Dhianpura, Roopnagar, \\ ${ }^{2}$ Punjab, Asst proff. Saraswati Nursing Institute, Dhianpura, Roopnagar, Punjab.
}

\begin{abstract}
Tuberculosis is one of the leading causes of morbidity and mortality around the world. Globally, the DOTS has been recognized as the best cost-effective approach for tuberculosis control and reduction of disease burden. The persons suffering from tuberculosis have difficulty in following a long-term treatment regimen. A non-experimental research approach was adopted for the study and exploratory research design was employed to explore the factors associated with adherence to DOTS therapy among tuberculosis patients. The Convenient Sampling technique was used to select the sample. The results of study showed that majority of the respondents $70 \%$ were adhered while $30 \%$ were non adhered to DOTS treatment. The findings revealed that area of residence and satisfaction with information received from health care personnel, were associated with adherence to DOTS therapy. Moreover, accessibility of health care facility, convenient TB center opening time, confidentiality issues, health personnel behavior, psychological stress due to continuation of DOTS therapy, relief from symptoms, difficulty in taking DOTS therapy and family support had also significant relationship with adherence to DOTS therapy while waiting time, paying money, language problems, supervision, side effects and social stigma were found to be non significant factors. The patients describes difficulties they were facing as long duration of treatment, quantity of pills, side effects and interference of DOTS therapy in daily routine. Thus, it implies that still, there is need of necessary interventions to reduce and eliminate the factors leading to non adherence as well as promotion of factors leading to adherence to DOTS therapy.
\end{abstract}

Keywords: Factors, Adherence, DOTS therapy, Tuberculosis Patients

\section{Introduction}

Tuberculosis is one of the leading causes of morbidity and mortality around the world. It is a disease caused by bacteria called as mycobacterium tuberculi that spreads through air. When a person suffering from pulmonary tuberculosis coughs or sneezes, millions of TB bacilli are spread in air embedded in the form of tiny droplets and droplet nuclei and infect another

person. ${ }^{1}$

The national tuberculosis program was started in 1962 in India with the aim to detect cases earliest and treat them. This program is operational in most of the districts replaced by DOTS strategy. When used properly, a course of DOTS therapy cures over $80 \%$ of patients. DOTS therapy has five elements beginning with political commitment which must be accompanied by sustained improvement for case detection, standardized treatment, reliable drug supply system, periodic evaluation and measurement. ${ }^{2}$

The qualities of diagnosis and treatment outcome in Tuberculosis had improved significantly with the introduction of DOTS. But still treatment adherence was a problem may be due to social stigma and deficient knowledge of the disease and its treatment. ${ }^{3}$

\section{Research Problem}

An Exploratory study to assess factors associated with adherence to DOTS therapy among patients reporting at selected DOTS centers in Punjab.

\section{Objectives}

1. To explore factors associated with adherence to DOTS therapy among tuberculosis patients.

2. To associate relationship between adherence to DOTS therapy with different socio- demographic variables.

\section{Research approach:}

\section{Material and Method}

A non experimental approach was used to explore factors associated with adherence to DOTS therapy among patients reporting at selected DOTS centers in Punjab. 


\section{Research design:}

An Exploratory research design was used to explore factors associated with adherence to DOTS therapy among patients reporting at selected DOTS centers in Punjab.

\section{Research setting:}

The present study was conducted in three DOTS centers - Primary Health Center Kurali, Community Health Center Fatehgarh Sahib and Ropar in Punjab.

Population: Tuberculosis patients

\section{Target Population:}

In present study it refers to all the patients who were suffering from tuberculosis and registered for DOTS therapy in selected DOTS centers in Punjab.

\section{Sample and Sampling Technique}

Sample

In this study, the sample of 100 tuberculosis patients who fulfilled the inclusion criteria, were included.

Sampling Technique:

Convenient sampling technique was used to collect the data.

\section{Sampling Criteria}

Inclusion Criteria

Patients suffering from tuberculosis who were

$>$ Reporting in selected DOTS centers in Punjab.

$>$ Receiving DOTS therapy.

$>$ Available at the time of data collection.

\section{Exclusion Criteria}

The patients who refused to participate in the study.

\section{Description of Tool}

TOOL 1:- It includes items related to demographic variables includes age in years, gender, educational status, religion, income per month, area of residence etc. and clinical profile of an individual.

TOOL II:-Standardized Drug Attitude Inventory rating scale is used to assess patient's adherence level to DOTS therapy.

Score interpretation-To calculate the score from a set of answers, each correct answer is given a score of plus one and each incorrect answer is given a score of minus one. The total score for each patient was calculated as the sum of the positive scores minus the negative scores. A positive total score indicates a positive subjective response (adherent) and a negative total scores indicates a negative subjective response (non adherent).

TOOL III:- A semi-structured interview schedule is used to explore factors associated with adherence to DOTS therapy among patients.

TABLE 1:- Frequency distribution of Adherent and Non adherent subjects according to Socio-demographic Variables.

\begin{tabular}{|c|c|c|c|c|c|c|}
\hline \multirow[t]{2}{*}{ Sr.No } & \multirow[t]{2}{*}{ DEMOGRAPHIC VARIABLE } & \multicolumn{2}{|c|}{ ADHERENT } & \multicolumn{2}{|c|}{ NON ADHERENT } & TOTAL \\
\hline & & $\mathbf{f}$ & $(\%)$ & & $(\%)$ & f $\quad(\%)$ \\
\hline 1. & $\begin{array}{l}\text { Age (in years) } \\
\leq 20 \\
21-40 \\
41-59 \\
\geq 60\end{array}$ & $\begin{array}{l}07 \\
42 \\
18 \\
03\end{array}$ & $\begin{array}{l}(10 \%) \\
(60 \%) \\
(26 \%) \\
(04 \%)\end{array}$ & $\begin{array}{l}02 \\
19 \\
08 \\
01\end{array}$ & $\begin{array}{l}(07 \%) \\
(63 \%) \\
(27 \%) \\
(03 \%)\end{array}$ & $\begin{array}{ll}09 & (09 \%) \\
61 & (61 \%) \\
26 & (26 \%) \\
04 & (04 \%) \\
\end{array}$ \\
\hline 2. & $\begin{array}{l}\text { Gender } \\
\text { Male } \\
\text { Female } \\
\end{array}$ & $\begin{array}{l}25 \\
45 \\
\end{array}$ & $\begin{array}{l}(36 \%) \\
(64 \%) \\
\end{array}$ & $\begin{array}{l}10 \\
20 \\
\end{array}$ & $\begin{array}{l}(33 \%) \\
(67 \%) \\
\end{array}$ & $\begin{array}{ll}35 & (35 \%) \\
65 & (65 \%) \\
\end{array}$ \\
\hline 3. & $\begin{array}{l}\text { Education } \\
\text { No formal education } \\
\text { Primary education } \\
\text { High school education } \\
\text { Graduation or above }\end{array}$ & $\begin{array}{l}04 \\
19 \\
27 \\
20\end{array}$ & $\begin{array}{l}(06 \%) \\
(27 \%) \\
(39 \%) \\
(28 \%)\end{array}$ & $\begin{array}{l}01 \\
10 \\
05 \\
14\end{array}$ & $\begin{array}{l}(03 \%) \\
(33 \%) \\
(17 \%) \\
(47 \%) \\
\end{array}$ & $\begin{array}{ll}05 & (05 \%) \\
29 & (29 \%) \\
32 & (32 \%) \\
34 & (34 \%) \\
\end{array}$ \\
\hline 4. & $\begin{array}{l}\text { Employment status } \\
\text { Employed } \\
\text { Unemployed } \\
\text { Retired } \\
\text { Student } \\
\end{array}$ & $\begin{array}{l}36 \\
25 \\
03 \\
06 \\
\end{array}$ & $\begin{array}{l}(51 \%) \\
(36 \%) \\
(04 \%) \\
(09 \%) \\
\end{array}$ & $\begin{array}{l}12 \\
16 \\
00 \\
02\end{array}$ & $\begin{array}{l}(40 \%) \\
(53 \%) \\
(00 \%) \\
(07 \%) \\
\end{array}$ & $\begin{array}{ll}48 & (48 \%) \\
41 & (41 \%) \\
03 & (03 \%) \\
08 & (08 \%) \\
\end{array}$ \\
\hline 5. & $\begin{array}{l}\text { Marital Status } \\
\text { Married } \\
\text { Unmarried }\end{array}$ & & $\begin{array}{l}(67 \%) \\
(33 \%)\end{array}$ & $\begin{array}{l}21 \\
09 \\
\end{array}$ & $\begin{array}{l}(70 \%) \\
(30 \%) \\
\end{array}$ & $\begin{array}{ll}68 & (68 \%) \\
32 & (32 \%) \\
\end{array}$ \\
\hline
\end{tabular}


An Exploratory Study to Assess Factors Associated with Adherence to Dots Therapy Among...

\begin{tabular}{|c|c|c|c|c|c|c|c|}
\hline 6. & $\begin{array}{l}\text { Income per month } \\
\text { (in rs.) } \\
\leq 5000 \\
5001-10,000 /- \\
10,001-20,000 /- \\
\geq 21,000\end{array}$ & $\begin{array}{l}34 \\
17 \\
10 \\
09 \\
\end{array}$ & $\begin{array}{l}(48 \%) \\
(25 \%) \\
(14 \%) \\
(13 \%) \\
\end{array}$ & & $\begin{array}{l}(53 \%) \\
(24 \%) \\
(03 \%) \\
(20 \%) \\
\end{array}$ & $\begin{array}{l}50 \\
24 \\
11 \\
15 \\
\end{array}$ & $\begin{array}{l}(50 \%) \\
(24 \%) \\
(11 \%) \\
(15 \%) \\
\end{array}$ \\
\hline \multirow[t]{2}{*}{ Sr.No } & \multirow[t]{2}{*}{ DEMOGRAPHIC VARIABLE } & \multicolumn{2}{|c|}{ ADHERENT } & \multicolumn{2}{|c|}{ NON ADHERENT } & \multicolumn{2}{|c|}{ TOTAL } \\
\hline & & $\mathbf{f}$ & $(\%)$ & & $(\%)$ & & $(\%)$ \\
\hline 7. & $\begin{array}{l}\text { Religion } \\
\text { Hindu } \\
\text { Muslim } \\
\text { Sikh } \\
\text { Christian }\end{array}$ & $\begin{array}{l}32 \\
17 \\
12 \\
09\end{array}$ & $\begin{array}{l}(46 \%) \\
(24 \%) \\
(17 \%) \\
(13 \%)\end{array}$ & $\begin{array}{l}10 \\
05 \\
08 \\
07\end{array}$ & $\begin{array}{l}(33 \%) \\
(17 \%) \\
(27 \%) \\
(23 \%)\end{array}$ & $\begin{array}{l}42 \\
22 \\
20 \\
16 \\
\end{array}$ & $\begin{array}{l}(42 \%) \\
(22 \%) \\
(20 \%) \\
(16 \%) \\
\end{array}$ \\
\hline 8. & $\begin{array}{l}\text { Area of residence } \\
\text { Rural } \\
\text { Urban }\end{array}$ & $\begin{array}{l}21 \\
49\end{array}$ & $\begin{array}{l}(30 \%) \\
(70 \%)\end{array}$ & $\begin{array}{l}19 \\
11\end{array}$ & $\begin{array}{l}(63 \%) \\
(37 \%)\end{array}$ & $\begin{array}{l}40 \\
60\end{array}$ & $\begin{array}{l}(40 \%) \\
(60 \%)\end{array}$ \\
\hline 9. & $\begin{array}{l}\text { Type of Family } \\
\text { Joint family } \\
\text { Nuclear family } \\
\end{array}$ & $\begin{array}{l}20 \\
50 \\
\end{array}$ & $\begin{array}{l}(29 \%) \\
(71 \%)\end{array}$ & $\begin{array}{l}13 \\
17 \\
\end{array}$ & $\begin{array}{l}(43 \%) \\
(57 \%)\end{array}$ & $\begin{array}{l}33 \\
67 \\
\end{array}$ & $\begin{array}{l}(33 \%) \\
(67 \%) \\
\end{array}$ \\
\hline 10. & $\begin{array}{l}\text { Source of information } \\
\text { ASHA worker } \\
\text { Television/Radio } \\
\text { Newspaper } \\
\text { Health professional } \\
\text { Any other } \\
\end{array}$ & $\begin{array}{l}02 \\
10 \\
32 \\
15 \\
11 \\
\end{array}$ & $\begin{array}{l}(03 \%) \\
(14 \%) \\
(46 \%) \\
(21 \%) \\
(16 \%)\end{array}$ & $\begin{array}{l}02 \\
08 \\
09 \\
05 \\
06\end{array}$ & $\begin{array}{l}(07 \%) \\
(27 \%) \\
(30 \%) \\
(16 \%) \\
(20 \%) \\
\end{array}$ & $\begin{array}{l}04 \\
18 \\
41 \\
20 \\
17\end{array}$ & $\begin{array}{l}(04 \%) \\
(18 \%) \\
(41 \%) \\
(20 \%) \\
(17 \%)\end{array}$ \\
\hline 11. & $\begin{array}{l}\text { Satisfaction with information received from } \\
\text { health care personnel regarding DOTS therapy } \\
\text { Excellent } \\
\text { Good } \\
\text { Unsatisfactory }\end{array}$ & $\begin{array}{l}19 \\
46 \\
05\end{array}$ & $\begin{array}{l}(27 \%) \\
(66 \%) \\
(07 \%)\end{array}$ & $\begin{array}{l}06 \\
14 \\
10\end{array}$ & $\begin{array}{l}(20 \%) \\
(47 \%) \\
(33 \%)\end{array}$ & $\begin{array}{l}25 \\
60 \\
15\end{array}$ & $\begin{array}{l}(25 \%) \\
(60 \%) \\
(15 \%)\end{array}$ \\
\hline 12. & $\begin{array}{l}\text { History of Associated diseases } \\
\text { Hypertension } \\
\text { Diabetes Mellitus } \\
\text { Any other } \\
\text { None }\end{array}$ & $\begin{array}{l}10 \\
07 \\
04 \\
49\end{array}$ & $\begin{array}{l}(14 \%) \\
(10 \%) \\
(06 \%) \\
(70 \%)\end{array}$ & $\begin{array}{l}04 \\
03 \\
07 \\
16 \\
\end{array}$ & $\begin{array}{l}(13 \%) \\
(10 \%) \\
(24 \%) \\
(53 \%)\end{array}$ & $\begin{array}{l}14 \\
10 \\
11 \\
65\end{array}$ & $\begin{array}{l}(14 \%) \\
(10 \%) \\
(11 \%) \\
(65 \%) \\
\end{array}$ \\
\hline
\end{tabular}

TABLE 2:- Frequency distribution of subjects regarding adherence to treatment regimen for the management of tuberculosis.

$(\mathrm{N}=100)$

\begin{tabular}{|l|l|cc|c|c|}
\hline \multicolumn{1}{|c|}{ Score } & Adherence category & $\mathbf{f}$ & $\mathbf{( \% )}$ & Mean & Standard Deviation \\
\hline Positive scores & Adherent & 70 & $(70 \%)$ & 17.90 & 4.081 \\
\hline Negative scores & Non adherent & 30 & $(30 \%)$ & 12.08 & 4.057 \\
\hline
\end{tabular}

Table depicts that majority of the subjects i.e. $70(70 \%)$ were adherent to DOTS therapy where as 30 (30\%) subjects were non adherent to the treatment regimen for the management of tuberculosis.

TABLE 3:-Frequency and Percentage distribution of all subjects regarding Health care facility related factors.

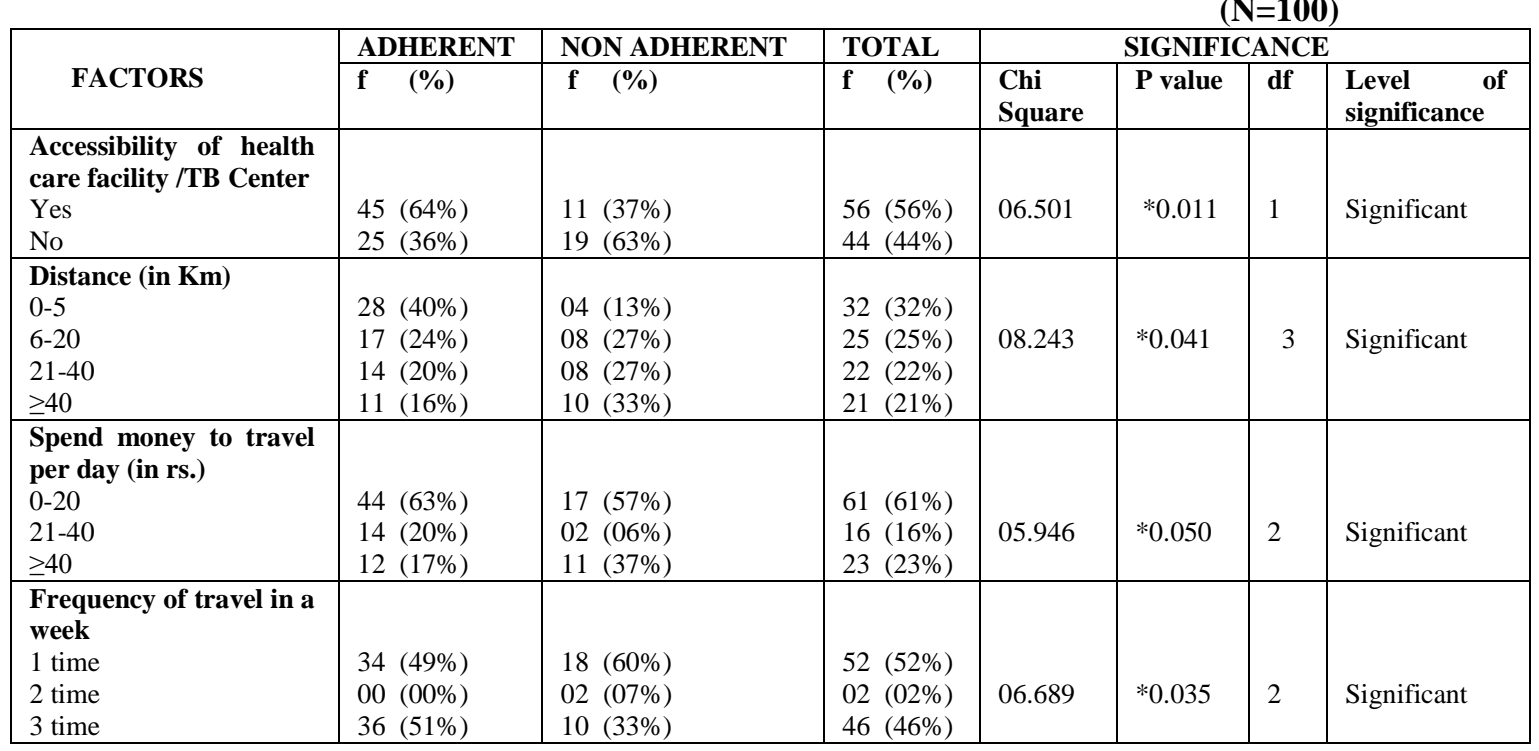


An Exploratory Study to Assess Factors Associated with Adherence to Dots Therapy Among...

\begin{tabular}{|l|l|l|l|l|l|l|}
\hline $\begin{array}{l}\text { Time taken to travel to } \\
\text { TB Center }\end{array}$ & & & & & \\
$0-15$ minutes & $39(56 \%)$ & $05(17 \%)$ & $44(44 \%)$ & & \\
$16-30$ minutes & $11(16 \%)$ & $05(17 \%)$ & $16(16 \%)$ & 17.176 & $* 0.001$ & 3 \\
$31-45$ minutes & $08(11 \%)$ & $04(13 \%)$ & $12(12 \%)$ & & Significant \\
$>45$ & $12(17 \%)$ & $16(53 \%)$ & $28(28 \%)$ & & \\
\hline
\end{tabular}

*(Significant Level $\leq 0.05)$

TABLE 4:- Frequency and percentage distribution of all subjects according to Health care facility related factors.

\begin{tabular}{|c|c|c|c|c|c|c|c|}
\hline \multirow[b]{2}{*}{ FACTORS } & ADHERENT & NON ADHERENT & TOTAL & \multicolumn{4}{|c|}{ SIGNIFICANCE } \\
\hline & f $\quad(\%)$ & f $(\%)$ & f $(\%)$ & $\begin{array}{l}\text { Chi } \\
\text { Square }\end{array}$ & $\begin{array}{l}\mathbf{P} \\
\text { value }\end{array}$ & df & $\begin{array}{l}\text { Level of } \\
\text { significance }\end{array}$ \\
\hline $\begin{array}{l}\text { Diagnostic Evaluation } \\
\text { before initiation of DOTS } \\
\text { therapy } \\
\text { Yes }\end{array}$ & $70(100 \%)$ & $30(100 \%)$ & $100(100 \%)$ & - & - & - & - \\
\hline $\begin{array}{l}\text { Waiting time at DOTS } \\
\text { Center } \\
\text { Yes }\end{array}$ & $19(27 \%)$ & $10(33 \%)$ & $29(29 \%)$ & & & & \\
\hline $\begin{array}{l}\text { a) } \leq 30 \text { minutes } \\
\text { b) }>30 \text { minutes }\end{array}$ & $\begin{array}{l}14(74 \%) \\
05(26 \%)\end{array}$ & $\begin{array}{l}07(70 \%) \\
03(30 \%)\end{array}$ & $\begin{array}{l}21(72 \%) \\
08(28 \%)\end{array}$ & 0.391 & 0.532 & 1 & $\begin{array}{l}\text { Non } \\
\text { significant }\end{array}$ \\
\hline No & $51(73 \%)$ & $20(67 \%)$ & $71(71 \%)$ & & & & \\
\hline $\begin{array}{l}\text { Pay money for DOTS } \\
\text { therapy } \\
\text { No }\end{array}$ & $70(100 \%)$ & $30(100 \%)$ & $100(100 \%)$ & - & - & - & - \\
\hline $\begin{array}{l}\text { Convenient TB Centre } \\
\text { opening time } \\
\text { Morning } \\
\text { Afternoon } \\
\text { Evening }\end{array}$ & $\begin{array}{l}37(53 \%) \\
17(24 \%) \\
16(23 \%) \\
\end{array}$ & $\begin{array}{l}10(33 \%) \\
15(50 \%) \\
05(17 \%) \\
\end{array}$ & $\begin{array}{l}47(47 \%) \\
32(32 \%) \\
21(21 \%) \\
\end{array}$ & 6.426 & $* 0.040$ & 2 & Significant \\
\hline $\begin{array}{l}\text { Availability of medicines } \\
\text { at DOTS Center } \\
\text { Always available }\end{array}$ & $70(100 \%)$ & $30(100 \%)$ & $100(100 \%)$ & - & - & - & - \\
\hline
\end{tabular}

$*$ (Significant Level $\leq 0.05)$

TABLE 5:- Frequency and percentage distribution of Adherent and Non-adherent subjects regarding Health care team related factors.

$(\mathrm{N}=\mathbf{1 0 0})$

\begin{tabular}{|c|c|c|c|c|c|c|c|}
\hline \multirow[b]{2}{*}{ FACTORS } & \multirow{2}{*}{$\begin{array}{l}\text { ADHERENT } \\
\text { f }(\%)\end{array}$} & \multirow{2}{*}{$\begin{array}{l}\text { NON ADHERENT } \\
\text { f }(\%)\end{array}$} & \multirow{2}{*}{$\frac{\text { TOTAL }}{\text { f }(\%)}$} & \multicolumn{4}{|c|}{ SIGNIFICANCE } \\
\hline & & & & $\begin{array}{l}\text { Chi } \\
\text { Square }\end{array}$ & $\begin{array}{l}\mathbf{p} \\
\text { value }\end{array}$ & df & $\begin{array}{l}\text { Level of } \\
\text { significance }\end{array}$ \\
\hline $\begin{array}{l}\text { Confidentiality } \\
\text { Issues } \\
\text { Yes } \\
\text { No }\end{array}$ & $\begin{array}{l}21(30 \%) \\
49(70 \%)\end{array}$ & $\begin{array}{l}18(60 \%) \\
12(40 \%)\end{array}$ & $\begin{array}{l}39(39 \%) \\
61(61 \%)\end{array}$ & 07.945 & $* 0.005$ & 1 & Significant \\
\hline $\begin{array}{l}\text { Language Problems } \\
\text { Yes } \\
\text { No }\end{array}$ & $\begin{array}{l}07(10 \%) \\
63(90 \%) \\
\end{array}$ & $\begin{array}{l}05(17 \%) \\
25(83 \%)\end{array}$ & $\begin{array}{l}12(12 \%) \\
88(88 \%)\end{array}$ & 00.884 & 0.347 & 1 & $\begin{array}{l}\text { Non } \\
\text { significant }\end{array}$ \\
\hline $\begin{array}{l}\text { Supervision while } \\
\text { taking DOTS doses } \\
\text { Yes } \\
\text { No }\end{array}$ & $\begin{array}{l}54(77 \%) \\
16(23 \%)\end{array}$ & $\begin{array}{l}26(87 \%) \\
04(13 \%)\end{array}$ & $\begin{array}{l}80(80 \%) \\
20(20 \%)\end{array}$ & 01.190 & 0.275 & 1 & $\begin{array}{l}\text { Non } \\
\text { significant }\end{array}$ \\
\hline $\begin{array}{l}\text { Health Personnel } \\
\text { Behaviour } \\
\text { Very friendly } \\
\text { Friendly } \\
\text { Unfriendly }\end{array}$ & $\begin{array}{l}11(16 \%) \\
35(50 \%) \\
24(34 \%)\end{array}$ & $\begin{array}{l}15(50 \%) \\
10(33 \%) \\
05(17 \%)\end{array}$ & $\begin{array}{l}26(26 \%) \\
45(45 \%) \\
29(29 \%)\end{array}$ & 13.039 & $* 0.001$ & 2 & Significant \\
\hline
\end{tabular}

*(Significant Level $\leq$ 0.05) 
An Exploratory Study to Assess Factors Associated with Adherence to Dots Therapy Among...

TABLE 6:- Frequency and percentage distribution of all subjects according to Treatment related factors

$(\mathbf{N}=\mathbf{1 0 0})$

\begin{tabular}{|c|c|c|c|c|c|c|c|}
\hline \multirow[b]{2}{*}{ FACTORS } & ADHERENT & NON ADHERENT & TOTAL & \multicolumn{4}{|c|}{ SIGNIFICANCE } \\
\hline & f $\quad(\%)$ & f $(\%)$ & f $(\%)$ & $\begin{array}{l}\text { Chi } \\
\text { Square }\end{array}$ & $\begin{array}{l}\mathbf{P} \\
\text { value }\end{array}$ & df & $\begin{array}{l}\text { Level of } \\
\text { significance }\end{array}$ \\
\hline $\begin{array}{l}\text { Classification of TB } \\
\text { New sputum positive case } \\
\text { Treatment after default } \\
\text { Failure }\end{array}$ & $\begin{array}{ll}58 & (82 \%) \\
10 & (15 \%) \\
02 & (03 \%) \\
\end{array}$ & $\begin{array}{ll}17 & (57 \%) \\
05 & (17 \%) \\
08 & (26 \%) \\
\end{array}$ & $\begin{array}{l}75(75 \%) \\
15(15 \%) \\
10(10 \%) \\
\end{array}$ & 13.905 & $* 0.001$ & 2 & Significant \\
\hline $\begin{array}{l}\text { Phase of DOTS treatment } \\
\text { Intensive phase } \\
\text { Continuation phase }\end{array}$ & $\begin{array}{ll}13 & (19 \%) \\
57 & (81 \%) \\
\end{array}$ & $\begin{array}{ll}02 & (07 \%) \\
28 & (93 \%) \\
\end{array}$ & $\begin{array}{l}15(15 \%) \\
85(85 \%)\end{array}$ & 2.334 & 0.127 & 1 & Non significant \\
\hline $\begin{array}{l}\text { Category of DOTS therapy } \\
\text { Category } 1 \\
\text { Category } 2 \\
\text { Category } 4\end{array}$ & $\begin{array}{ll}58 & (83 \%) \\
10 & (14 \%) \\
02 & (03 \%)\end{array}$ & $\begin{array}{ll}18 & (60 \%) \\
05 & (17 \%) \\
07 & (23 \%) \\
\end{array}$ & $\begin{array}{l}76(76 \%) \\
15(15 \%) \\
09(09 \%)\end{array}$ & 11.306 & $* 0.004$ & 2 & Significant \\
\hline $\begin{array}{l}\text { Full information about } \\
\text { DOTS is provided by health } \\
\text { personnel } \\
\text { Yes } \\
\text { No }\end{array}$ & $\begin{array}{ll}50 & (71 \%) \\
20 & (29 \%)\end{array}$ & $\begin{array}{ll}10 & (33 \%) \\
20 & (67 \%)\end{array}$ & $\begin{array}{l}60(60 \%) \\
40(40 \%)\end{array}$ & 12.698 & $* 0.000$ & 1 & Significant \\
\hline $\begin{array}{l}\text { DOTS interfering in daily } \\
\text { activities } \\
\text { Yes } \\
\text { No }\end{array}$ & $\begin{array}{ll}38 & (54 \%) \\
32 & (46 \%)\end{array}$ & $\begin{array}{ll}27 & (90 \%) \\
03 & (10 \%)\end{array}$ & $\begin{array}{l}65(65 \%) \\
35(35 \%)\end{array}$ & 11.774 & $* 0.001$ & 1 & Significant \\
\hline
\end{tabular}

*(Significant Level $\leq \mathbf{0 . 0 5})$

TABLE 8:- Frequency and percentage distribution of Adherent and Non-adherent subjects according to Treatment related factors.

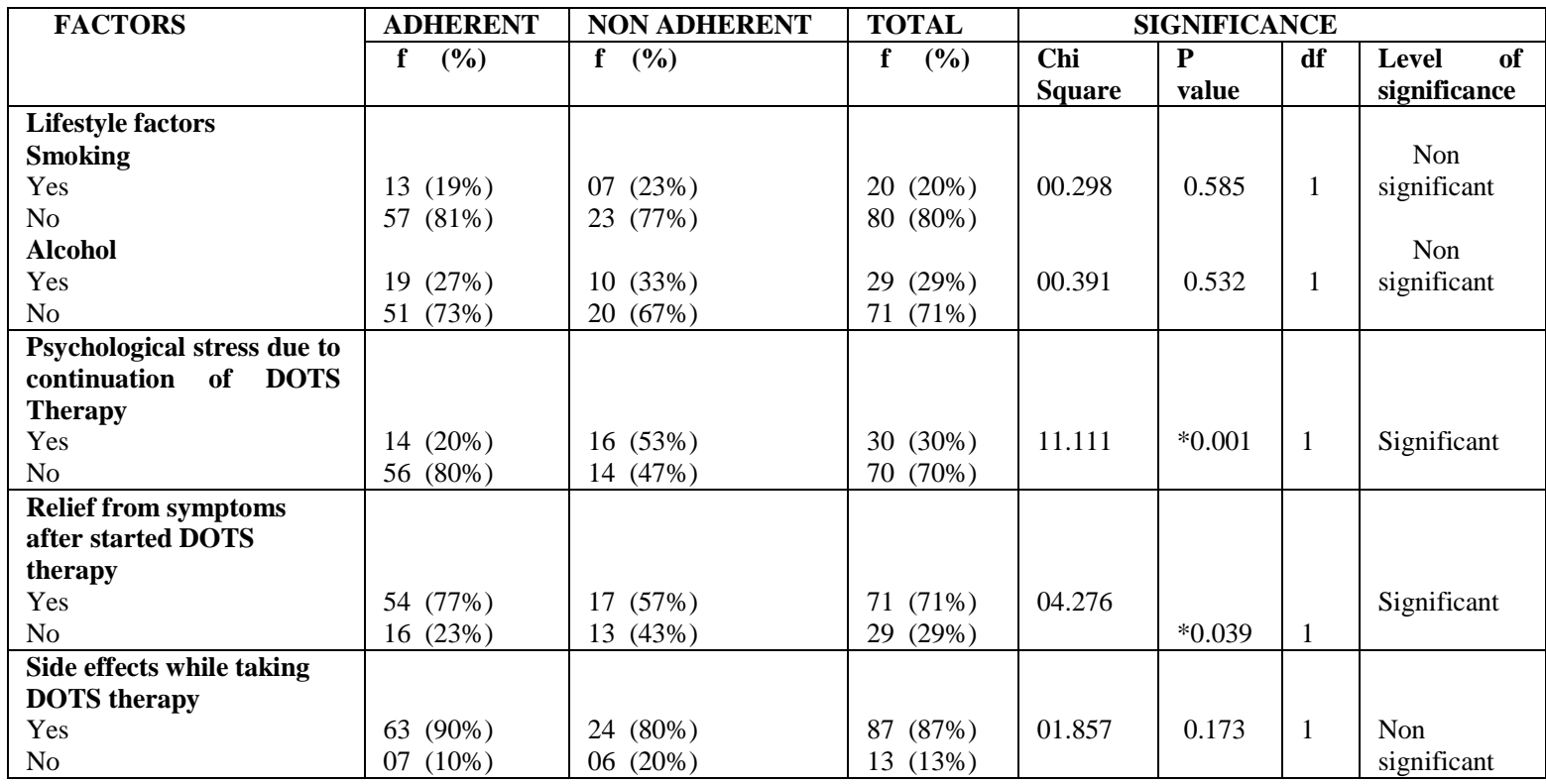

$*$ (Significant Level $\leq \mathbf{0 . 0 5})$

TABLE 9:- Frequency and percentage distribution of Adherent and Non-adherent groups according to community related factors.

$(\mathrm{N}=\mathbf{1 0 0})$

\begin{tabular}{|c|c|c|c|c|c|c|c|}
\hline \multirow[b]{2}{*}{ FACTORS } & ADHERENT & NON ADHERENT & TOTAL & \multicolumn{4}{|c|}{ SIGNIFICANCE } \\
\hline & f $(\%)$ & f $(\%)$ & f $(\%)$ & $\begin{array}{l}\text { Chi } \\
\text { Square }\end{array}$ & $\begin{array}{l}\mathbf{P} \\
\text { value }\end{array}$ & df & $\begin{array}{l}\text { Level of } \\
\text { significance }\end{array}$ \\
\hline $\begin{array}{l}\text { Length of stay at } \\
\text { current place } \\
<6 \text { months } \\
6-12 \text { months } \\
>12 \text { months }\end{array}$ & $\begin{array}{l}07(10 \%) \\
18(26 \%) \\
45(64 \%)\end{array}$ & $\begin{array}{l}03(10 \%) \\
10(33 \%) \\
17(57 \%)\end{array}$ & $\begin{array}{l}10(10 \%) \\
28(28 \%) \\
62(62 \%)\end{array}$ & 00.632 & 0.729 & 2 & $\begin{array}{c}\text { Non } \\
\text { significant }\end{array}$ \\
\hline
\end{tabular}


An Exploratory Study to Assess Factors Associated with Adherence to Dots Therapy Among...

\begin{tabular}{|c|c|c|c|c|c|c|c|}
\hline $\begin{array}{l}\text { Disclosed } \\
\text { status to friends/ } \\
\text { relatives } \\
\text { Yes } \\
\text { No }\end{array}$ & $\begin{array}{l}27(39 \%) \\
43(61 \%)\end{array}$ & $\begin{array}{l}09(30 \%) \\
21(70 \%)\end{array}$ & $\begin{array}{l}36(36 \%) \\
64(64 \%)\end{array}$ & 00.670 & 0.413 & 1 & $\begin{array}{l}\text { Non } \\
\text { significant }\end{array}$ \\
\hline $\begin{array}{l}\text { Facing social } \\
\text { stigma due to TB } \\
\text { Yes } \\
\text { No }\end{array}$ & $\begin{array}{l}23(33 \%) \\
47(67 \%) \\
\end{array}$ & $\begin{array}{l}11(37 \%) \\
19(63 \%)\end{array}$ & $\begin{array}{l}34(34 \%) \\
66(66 \%) \\
\end{array}$ & 00.136 & 0.712 & 1 & $\begin{array}{l}\text { Non } \\
\text { significant }\end{array}$ \\
\hline $\begin{array}{l}\text { Changes in } \\
\text { behavior of family } \\
\text { and friends } \\
\text { Yes } \\
\text { No }\end{array}$ & $\begin{array}{l}24(34 \%) \\
46(66 \%)\end{array}$ & $\begin{array}{l}11(37 \%) \\
19(63 \%)\end{array}$ & $\begin{array}{l}35(35 \%) \\
65(65 \%)\end{array}$ & 00.052 & 0.819 & 1 & $\begin{array}{l}\text { Non } \\
\text { significant }\end{array}$ \\
\hline $\begin{array}{l}\text { Family Support } \\
\text { Yes } \\
\text { No }\end{array}$ & $\begin{array}{l}54(77 \%) \\
16(23 \%)\end{array}$ & $\begin{array}{l}0930 \%) \\
21(70 \%)\end{array}$ & $\begin{array}{l}63(63 \%) \\
37(37 \%)\end{array}$ & 20.022 & $\begin{array}{l}* 0.00 \\
0\end{array}$ & 1 & Significant \\
\hline
\end{tabular}

*(Significant Level $\leq \mathbf{0 . 0 5})$

TABLE 10 :- Association between socio- demographic variables and adherence to DOTS therapy among patients suffering from tuberculosis.

\begin{tabular}{|c|c|c|c|c|c|c|c|}
\hline \multirow{3}{*}{$\begin{array}{l}\text { DEMOGRAPHIC } \\
\text { VARIABLE }\end{array}$} & \multirow{3}{*}{$\begin{array}{l}\text { ADHERENT } \\
\text { f }(\%)\end{array}$} & \multirow{3}{*}{\begin{tabular}{|l} 
NON ADHERENT \\
f $(\%)$
\end{tabular}} & \multirow{2}{*}{ TOTAL } & & \multicolumn{3}{|c|}{$(\mathrm{N}=100)$} \\
\hline & & & & \multicolumn{4}{|c|}{ SIGNIFICANCE } \\
\hline & & & f $(\%)$ & $\begin{array}{l}\text { Chi } \\
\text { Square }\end{array}$ & $\begin{array}{l}P \\
\text { value }\end{array}$ & df & $\begin{array}{l}\text { Level of } \\
\text { significance }\end{array}$ \\
\hline $\begin{array}{l}\text { Age (in years) } \\
\leq 20 \\
21-40 \\
41-59 \\
\geq 60\end{array}$ & $\begin{array}{l}07(10 \%) \\
42(60 \%) \\
18(26 \%) \\
03(04 \%)\end{array}$ & $\begin{array}{l}02(07 \%) \\
19(63 \%) \\
08(27 \%) \\
01(03 \%)\end{array}$ & $\begin{array}{l}09(09 \%) \\
61(61 \%) \\
26(26 \%) \\
04(04 \%)\end{array}$ & 0.352 & 0.950 & 3 & $\begin{array}{c}\text { Non } \\
\text { significant }\end{array}$ \\
\hline $\begin{array}{l}\text { Gender } \\
\text { Male } \\
\text { Female } \\
\end{array}$ & $\begin{array}{l}25(36 \%) \\
45(64 \%) \\
\end{array}$ & $\begin{array}{l}10(33 \%) \\
20(67 \%)\end{array}$ & $\begin{array}{l}35(35 \%) \\
65(65 \%) \\
\end{array}$ & 0.052 & 0.819 & 1 & $\begin{array}{c}\text { Non } \\
\text { Significant }\end{array}$ \\
\hline $\begin{array}{l}\text { Education } \\
\text { No formal education } \\
\text { Primary education } \\
\text { High school education } \\
\text { Graduation or above }\end{array}$ & $\begin{array}{l}04(06 \%) \\
19(27 \%) \\
27(39 \%) \\
20(28 \%)\end{array}$ & $\begin{array}{l}01(03 \%) \\
10(33 \%) \\
05(17 \%) \\
14(47 \%)\end{array}$ & $\begin{array}{l}05(05 \%) \\
29(29 \%) \\
32(32 \%) \\
34(34 \%)\end{array}$ & 5.687 & 0.128 & 3 & $\begin{array}{c}\text { Non } \\
\text { Significant }\end{array}$ \\
\hline $\begin{array}{l}\text { Employment status } \\
\text { Employed } \\
\text { Unemployed } \\
\text { Retired } \\
\text { Student } \\
\end{array}$ & $\begin{array}{l}36(51 \%) \\
25(36 \%) \\
03(04 \%) \\
06(09 \%) \\
\end{array}$ & $\begin{array}{l}12(40 \%) \\
16(53 \%) \\
00(00 \%) \\
02(07 \%) \\
\end{array}$ & $\begin{array}{l}48(48 \%) \\
41(41 \%) \\
03(03 \%) \\
08(08 \%)\end{array}$ & 3.542 & 0.315 & 3 & $\begin{array}{c}\text { Non } \\
\text { Significant }\end{array}$ \\
\hline $\begin{array}{l}\text { Income per month } \\
\text { (in rs.) } \\
\leq 5000 \\
5001-10,000 /- \\
10,001-20,000 /- \\
\geq 21,000\end{array}$ & $\begin{array}{l}34(48 \%) \\
17(25 \%) \\
10(14 \%) \\
09(13 \%)\end{array}$ & $\begin{array}{l}16(53 \%) \\
07(24 \%) \\
01(03 \%) \\
06(20 \%)\end{array}$ & $\begin{array}{l}50(50 \%) \\
24(24 \%) \\
11(11 \%) \\
15(15 \%)\end{array}$ & 3.108 & 0.375 & 3 & $\begin{array}{c}\text { Non } \\
\text { Significant }\end{array}$ \\
\hline DEMOGRAPHIC & ADHERENT & NON ADHERENT & TOTAL & & SIG & IFI & NCE \\
\hline & f $(\%)$ & f $\quad(\%)$ & f $\quad(\%)$ & $\begin{array}{l}\text { Chi } \\
\text { Square }\end{array}$ & $\begin{array}{l}\mathbf{P} \\
\text { value }\end{array}$ & df & $\begin{array}{l}\text { Level of } \\
\text { significance }\end{array}$ \\
\hline $\begin{array}{l}\text { Marital Status } \\
\text { Married } \\
\text { Unmarried }\end{array}$ & $\begin{array}{l}47(67 \%) \\
23(33 \%)\end{array}$ & $\begin{array}{l}21(70 \%) \\
09(30 \%)\end{array}$ & $\begin{array}{l}68(68 \%) \\
32(32 \%)\end{array}$ & 0.079 & 0.779 & 1 & $\begin{array}{c}\text { Non } \\
\text { Significant }\end{array}$ \\
\hline $\begin{array}{l}\text { Religion } \\
\text { Hindu } \\
\text { Muslim } \\
\text { Sikh } \\
\text { Christian } \\
\end{array}$ & $\begin{array}{l}32(46 \%) \\
17(24 \%) \\
12(17 \%) \\
09(13 \%) \\
\end{array}$ & $\begin{array}{l}10(33 \%) \\
05(17 \%) \\
08(27 \%) \\
07(23 \%) \\
\end{array}$ & $\begin{array}{l}42(42 \%) \\
22(22 \%) \\
20(20 \%) \\
16(16 \%)\end{array}$ & 3.713 & 0.294 & 3 & $\begin{array}{c}\text { Non } \\
\text { Significant }\end{array}$ \\
\hline $\begin{array}{l}\text { Area of residence } \\
\text { Rural } \\
\text { Urban }\end{array}$ & $\begin{array}{l}21(30 \%) \\
49(70 \%)\end{array}$ & $\begin{array}{l}19(63 \%) \\
11(37 \%) \\
\end{array}$ & $\begin{array}{l}40(40 \%) \\
60(60 \%) \\
\end{array}$ & 9.722 & $* 0.02$ & 1 & Significant \\
\hline $\begin{array}{l}\text { Type of Family } \\
\text { Joint family } \\
\text { Nuclear family }\end{array}$ & $\begin{array}{l}20(29 \%) \\
50(71 \%)\end{array}$ & $\begin{array}{l}13(43 \%) \\
17(57 \%)\end{array}$ & $\begin{array}{l}33(33 \%) \\
67(67 \%)\end{array}$ & 2.070 & 0.150 & 1 & $\begin{array}{c}\text { Non } \\
\text { Significant }\end{array}$ \\
\hline
\end{tabular}


An Exploratory Study to Assess Factors Associated with Adherence to Dots Therapy Among...

\begin{tabular}{|c|c|c|c|c|c|c|c|}
\hline $\begin{array}{l}\text { Source } \\
\text { information } \\
\text { ASHA worker } \\
\text { Television/Radio } \\
\text { Newspaper } \\
\text { Health professional } \\
\text { Any other }\end{array}$ & $\begin{array}{l}02(03 \%) \\
10(14 \%) \\
32(46 \%) \\
15(21 \%) \\
11(16 \%)\end{array}$ & $\begin{array}{l}02(07 \%) \\
08(27 \%) \\
09(30 \%) \\
05(16 \%) \\
06(20 \%)\end{array}$ & $\begin{array}{l}04(04 \%) \\
18(18 \%) \\
41(41 \%) \\
20(20 \%) \\
17(17 \%)\end{array}$ & 4.280 & 0.369 & 4 & $\begin{array}{c}\text { Non } \\
\text { Significant }\end{array}$ \\
\hline $\begin{array}{l}\text { Satisfaction with } \\
\text { information } \\
\text { Excellent } \\
\text { Good } \\
\text { Unsatisfactory } \\
\end{array}$ & $\begin{array}{l}19(27 \%) \\
46(66 \%) \\
05(07 \%)\end{array}$ & $\begin{array}{l}06(20 \%) \\
14(47 \%) \\
10(33 \%)\end{array}$ & $\begin{array}{l}25(25 \%) \\
60(60 \%) \\
15(15 \%)\end{array}$ & 11.302 & $\begin{array}{l}* 0.00 \\
4\end{array}$ & 2 & Significant \\
\hline $\begin{array}{l}\text { History of } \\
\text { Associated diseases } \\
\text { Hypertension } \\
\text { Diabetes Mellitus } \\
\text { Any other } \\
\text { None }\end{array}$ & $\begin{array}{l}10(14 \%) \\
07(10 \%) \\
04(06 \%) \\
49(70 \%)\end{array}$ & $\begin{array}{l}04(13 \%) \\
03(10 \%) \\
07(24 \%) \\
16(53 \%)\end{array}$ & $\begin{array}{l}14(14 \%) \\
10(10 \%) \\
11(11 \%) \\
65(65 \%) \\
\end{array}$ & 6.837 & 0.077 & 3 & $\begin{array}{c}\text { Non } \\
\text { Significant }\end{array}$ \\
\hline
\end{tabular}

*(Significant Level $\leq \mathbf{0 . 0 5})$

\section{Conclusion}

The findings of the study revealed that majority of patients were adhered while more than one fourth of the patients still non adhered. The accessibility of health care facility, convenient TB center opening time, confidentiality issues, health personnel behavior, psychological stress, relief from symptoms, difficulty in taking DOTS therapy and family support had also significant relationship with adherence while waiting time, paying money, language problems, supervision, side effects and social stigma were found to be non significant factors. Hence, there was a need to resolve the difficulties of tuberculosis patients to promote adherence level to DOTS therapy.

\section{Ethical Consideration}

- Written permission was taken from the higher authority of the selected DOTS centers of the Punjab.

- Informed consent was taken from each participants of the study.

- Confidentiality and privacy of the study subjects will also be taken care of.

Source of Funding: Self

\section{References}

[1]. Mohan P.; National Institute of Allergy and Infectious diseases ; 2006 Available from URL: http://www.Niaid.nih.gov

[2]. G. Maarten, RJ Wilkinson RJ; Tuberculosis ; Lancet 2007; 2030-2043; Pub Med Abstract Publisher Full Text

[3]. Singh AV, JA Ogden, Sharma PP, Sar Arora ,VK Jain ; "Adherence to tuberculosis treatment-lessons from the urban setting of Delhi, India"; Trop Med Int Health ; 2003 Jul;625-33 . 